\title{
Mesenchymal Stem Cells Prevent Ovariectomy- Induced Osteoporosis Formation in Mice by Intraosseous Vascular Remodeling
}

\section{Weizhou Wang}

Kunming Medical University

\section{Yanghao Wang}

Kunming Medical University

\section{Zhihong Tang}

Kunming Medical University

\section{Yongcheng Chen}

Kunming Medical University

\section{Zhui Liu}

Kunming Medical University

\section{Hao Duan}

Kunming Medical University First Affilliated Hospital

\section{Zongyu Zhong}

Kunming Medical University First Affilliated Hospital

Fei He ( $\nabla$ drhefei@126.com )

Kunming Medical University First Affilliated Hospital https://orcid.org/0000-0002-1681-0265

\section{Research}

Keywords: Postmenopausal osteoporosis, Bone marrow mesenchymal stem cell, Bone mass recovery, Revascularization, Cellular therapy

Posted Date: May 5th, 2021

DOl: https://doi.org/10.21203/rs.3.rs-461062/v1

License: (c) (i) This work is licensed under a Creative Commons Attribution 4.0 International License. Read Full License 


\section{Abstract}

\section{Background}

Postmenopausal osteoporosis is the most common type of primary osteoporosis that is characterized by a decrease in bone mass and an increase risk of fractures. Mesenchymal stem cells (MSCs) can promote osteogenesis and are a promising therapy for postmenopausal osteoporosis. The aim of this study was to clarify the relationship between improved intraosseous microcirculation and increased bone mass by MSCs in postmenopausal osteoporosis.

\section{Methods}

We isolated primary MSCs and characterized their cellular properties. Bone volume and morphological analyses of mouse femurs were performed by transplanting the cells into post-ovariectomized mice via tail vein. Femoral artery ligation was performed on mice. By Laser Speckle flowmetry, vascular perfusion and femoral trabecular bone and cortical bone analysis, we determined the effects of MSCs in promoting intraosseous angiogenesis and preventing osteoporosis in mice.

\section{Results}

MSCs transplantation enhanced trabecular number, trabecular bone volume / total volume, and trabecular bone mineral density in ovariectomized mice. Reduced intraosseous blood flow due to hindlimb ischemia can lead to accelerated bone loss. In contrast, MSCs promoted the reconstruction of intraosseous microcirculation and reduced bone loss.

\section{Conclusions}

MSCs could effectively prevent postmenopausal osteoporosis formation, which is associated with the involvement of MSCs in the reestablishment of microcirculation within the skeleton. This study has revealed the potential role of MSCs in the treatment of postmenopausal osteoporosis and lays the foundation for clinical application studies.

\section{Introduction}

Osteoporosis is an age-related degenerative systemic bone disease characterized by a reduce in bone mass. Osteoporosis often presents with reduced bone mineral density (BMD) and an increasing risk of fracture of the lumbar spine and femoral neck ${ }^{[1 ; 2]}$. With the aging of population in the contemporary world, the Individual and social costs of osteoporosis are rising year by year, which poses a huge challenge to public health care $^{[3]}$. The disease not only has a serious impact on the quality and health of life of the elderly, but also imposes a significant health burden on family and society ${ }^{[4]}$. At present, the treatment of osteoporosis is mainly drug therapy ${ }^{[5 ; 6]}$. The drugs with significant efficacy for the therapy of osteoporosis mainly include bone formation promoters, bone resorption inhibitors, and bone minerals. 
However, the presence of a series of side effects, such as gastric mucosal damage, muscle pain, and fever, has somewhat limited its clinical applications ${ }^{[7 ; 8]}$.

Postmenopausal osteoporosis is the most common type of primary osteoporosis, which is mainly due to estrogen deficiency. It has been reported that estrogen deficiency leads to an increasing of bone remodeling, shifting the balance between bone formation and resorption in favor of the latter, which leads to osteoporosis and pathologic fracture ${ }^{[9 ; 10]}$. It has been previously shown that the number and distribution of intraosseous vasculature plays an important role in bone turnover and regulating bone mass $^{[11]}$. Inadequate or decrease bone vascularity is associated with osteoporosis ${ }^{[12]}$. However, the improvement of vascularity and microenvironment within bone tissue has received little attention.

A subpopulation of bone marrow mesenchymal stem cells, also known as mesenchymal stem cells (MSCs), have been used to treat a number of diseases in clinic ${ }^{[13 ; 14]}$. MSCs transplantation (MSCT) is a potential therapy for osteoporosis, which could effectively treat age-related and hormone deficiencyinduced osteoporosis ${ }^{[15]}$. However, the mechanisms of MSCT in the therapy of osteoporosis have not been fully elucidated, thus limiting its clinical applications to a certain degree. Numerous studies have shown that MSCT not only has positive effects in improving myocardial ischemia ${ }^{[16]}$, cerebral ischemia ${ }^{[17]}$ and hindlimb ischemia ${ }^{[18]}$, but also promotes angiogenesis ${ }^{[15]}$. The question still remains on whether MSCT could reestablish intraosseous microcirculation, thereby maintaining the bone mass of the development of osteoporosis. Based on these findings, we hypothesized that MSCT could prevent bone loss in osteoporosis by promoting the reconstruction of intraosseous microcirculation. This study will explore the role of MSCT in postmenopausal osteoporosis and lay the foundation for further clinical application.

\section{Materials And Methods}

\subsection{Extraction and culture of mouse MSCs}

All mice experiments were inspected and approved by the Animal Experiment Ethics Review Committee of Kunming Medical University (20200059), and all procedures were carried out in accordance with the guidelines of the ethical review committee.

To isolate the primary mouse MSCs, C57BL/ 6 female mice aged 8 weeks (weight: $18-21 \mathrm{~g}$ ) were killed by cervical dislocation and immersed in $75 \%$ ethanol for $5 \mathrm{~min}$. Then, the mice femurs were rapidly separated out and clipped. The bone marrow cavity was repeatedly rinsed with sterile PBS (Gibco, USA), the filtered twice using a cell strainer, and the filtrate was obtained and centrifuged at $1500 \mathrm{rmp}$ for $10 \mathrm{~min}$. After removal of supernatant, the sediment was suspended in low glucose GlutaMAX ${ }^{\mathrm{TM}}$ Supplement of Dulbecco's Modified Eagle Medium (DMEM, Gibco, USA), supplemented with 15\% fetal bovine serum (FBS, Gibco, USA) and $100 \mathrm{U} / \mathrm{mL}$ penicillin-streptomycin (Gibco, USA). The Cell suspensions were cultured in $75 \mathrm{~cm}^{2}$ culture flask and placed in an incubator (ThermoFisher, USA) at $37^{\circ} \mathrm{C}$ and $5 \% \mathrm{CO}_{2}$. 
Change the culture solution once every 2-3 days. When there is $80 \%-90 \%$ cell confluence., they were passaged with TrypLE ${ }^{\mathrm{TM}}$ Express Enzyme (Gibco, USA) digestion. Early passage MSCs (passage 3-7) were used for experiments.

\subsection{Identification of mouse MSCs}

In order to clarify the differentiation abilities of the osteogenic, adipogenic, and chondrogenic induction was performed for MSCs. MSCs were cultured for up to 21 days in osteogenic medium (Cyagen, China), 14 days in adipogenic medium (Cyagen, China) and 28 days chondrogenic medium (Cyagen, China) to promote differentiation. The induced cells were then stained with Alizarin Red solution to verify osteogenic differentiation, with Oil Red-O solution to verify lipogenic differentiation and with Alcian Blue solution to verify chondrogenic differentiation. All the staining was observed and photographed under microscope (Nikon, Japan).

To further characterize the cells, the expression of surface markers of MSCs was analyzed by flow cytometry. MSCs at passage 3-5 were washed in PBS and then harvested by incubation in TrypLE ${ }^{\mathrm{TM}}$ Express Enzyme (Gibco, USA). After centrifugation, MSCs pellets were washed with PBS and unspecific binding sites were blocked using anti-mouse CD16/CD32 (BD, USA) for $10 \mathrm{~min}$. Afterwards, the cells were centrifuged under light-proof conditions and then treated with anti-CD11b PE (Biolegend, USA), anti-CD29 APC (Biolegend), anti-CD44 Pacific Blue (Biolegend, USA), anti-CD45 PE/Cyanine5 (Biolegend, USA), antiCD117 Alexa Fluor 700 (Biolegend, USA) and anti-Sca-1 PE/Cyanine7 (Biolegend, USA) were incubated for 30 min, then washed with PBS and resuspended. Detection was performed within $1 \mathrm{~h}$ using flow cytometry (BD FACSAria, USA). Instrument compensation was performed using microbeads coated with anti-rat/anti-hamster antibodies (BD CompBeads, USA) and their attached antibodies. Flow cytometry data were analyzed using Flow Jo software (version V10.0).

\subsection{Osteoporosis mice model and cell transplantation}

C57BL/6 mice (weight: 18-21 g), 6 to 8 weeks old, were purchased from the Experimental Animal Center of Kunming Medical University. The mice were housed under the indicated conditions (12 hours of light and 12 hours of darkness, temperature $19-22^{\circ} \mathrm{C}$, humidity $55 \pm 5 \%$ ). The mice have free access to standard food and water.

To establish the osteoporosis model, mice were divided randomly into three groups consisting of 12 mice each: the sham group, the ovariectomy (OVX) group and the OVX \& MSCT group. Mice were anesthetized by intraperitoneal injection with $10 \%$ chloral hydrate (Kermel, China). After anaesthesia mice were fixed in prone position and the hair of dorsal skin was removed. Bilateral ovariectomy through the dorsal bilateral, the removed tissues include bilateral ovaries, ovarian envelope and part of the fallopian tubes, and then the incision was stapled and the skin was disinfected. In the sham group, a small amount of adipose tissue around the ovary was removed. The mice were placed on a thermostatic blanket during the surgery and postoperative recovery period until fully awake, and then the mice were housed in cages accommodating a normal diet. At day 7 and day 21 postoperatively, the mice of OVX \& MSCT group were infused MSCs at a dose of $1 \times 10^{6}$ per mouse by tail vein. The sham group and OVX group mice were 
infused an equivalent volume $(200 \mu \mathrm{l})$ of PBS. At day 35 post-surgery, all mice of three groups were sacrificed by dislocating their cervical spine. The hindlimb of the mice were extracted and the muscles and soft tissues attached to the bones were removed to obtain bilateral femurs. The mouse femurs were immersed in $4 \%$ paraformaldehyde at $4^{\circ} \mathrm{C}$ for $48 \mathrm{~h}$. The left femur bone was used to prepare histopathological sections, while the right femur was used for micro-CT and other tests.

\subsection{Femoral artery ligation mice model and cell transplantation}

To explore the effect of intraosseous vessels on femoral bone mass, all mice were exposed to OVX surgery as described. Afterwards, the mice were divided randomly into two groups consisting of 12 mice each: FAL group and FAL\& MSCT group. At day 7 after OVX surgery, the hindlimb ischemia model was created by the left femoral artery ligation (FAL).

The mice were anesthetized by intraperitoneal injection with $10 \%$ chloral hydrate and fixed in supine position on the operating table. Mice were excised from the hindlimbs bilaterally. Under sterile conditions, the left femoral artery was isolated and ligated with an 8 - 0 silk thread under an operating microscope (Leica, Germany). After disconnecting the left femoral artery, the incision was sutured and the skin was disinfected. The right hindlimb of mice was sham-operated group, only the arteries and surrounding soft tissues were exposed and separated, the femoral artery was not ligated. The mice were placed on a thermostatic blanket during the surgery and postoperative recovery period until fully awake, and then the mice were housed in cages accommodating a normal diet. MSCT was performed 24 hours and day 24 after ligation of the femoral artery for FAL \& MSCT groups. MSCs at a dose of $1 \times 10^{6}$ per mouse were infused by tail vein. The FAL group received equal volumes $(200 \mu \mathrm{l})$ of PBS by tail vein injections at the corresponding time points. At day 35 post FAL surgery, all mice of two groups were sacrificed by dislocating their cervical spine. The hindlimb of the mice were extracted and the muscles and soft tissues attached to the bones were removed to obtain bilateral femurs. The mouse femurs were immersed in $4 \%$ paraformaldehyde at $4^{\circ} \mathrm{C}$ for $48 \mathrm{~h}$. The femurs were used for micro-CT and immunofluorescence staining. Imaging of intraosseous vessels by perfusion of angiographic contrast in excess mice.

\subsection{Femoral micro-CT analysis}

Imaging and quantitative analysis of the mouse femurs using micro-CT (NEMO, China) followed a previously reported protocol ${ }^{[19]}$. The samples were placed in a transparent cylindrical recess and fixed with paper tape. The following detector parameters were set: the ray source tube voltage, $90 \mathrm{kV}$; electric current, $60 \mu \mathrm{A} ;$ frame rate, 20 . The scan and reconstruction were performed using Cruiser software, the data of region of interest ( $\mathrm{ROI}$ ) was analyzed using Avatar 3 software, and CT images were reconstructed using the FDK algorithm, CT view field of $15 \mathrm{~mm}$, pixel size of $0.0146 \mathrm{~mm}$, and slice thickness of $0.025 \mathrm{~mm}$. After reconstruction of the scanned data, ROI was selected for trabecular and cortical bone analysis according to the anatomical structure of those bone tissues. Trabecular bone analysis was selected $0.5 \mathrm{~mm}$ above the growth plate of the distal femur. Cortical bone parameter analysis was selected in the mid-diaphyseal region of the femur. The following analytical data were obtained: bone 
volume fraction (BV/TV), number (N), and BMD of the trabeculae (Tb); BMD, thickness (Th), and area fraction (Ar/Tt.Ar) of the cortical bone (Ct).

\subsection{Histological analysis and bone resorption detection}

After $4 \%$ paraformaldehyde fixed the femur for 48 hours, the femur decalcified by shaking with $0.5 \mathrm{M}$ Ethylene diamine tetraacetic acid (EDTA) (Solarbio, China) for 24-48h. After decalcification, the femurs were dehydrated, embedded in paraffin, and sectioned at $10 \mu \mathrm{m}$ thickness. Subsequently, the sections were dewaxed and hydrated, followed by HE staining, Masson staining and Trap staining. All staining was performed using the staining kit (Solarbio, China) according to the manufacturer instructions. The sections were dehydrated in gradient ethanol, then treated with xylene and sealed with neutral gum, and observed by light microscopy (Olympus, Japan) and photographed. Histological results were analyzed by ImageJ software (version 2.0). HE staining was used to observe morphological and histological changes. Masson staining was performed to observe the collagen fiber per bone surface (CF/BS) of the femur metaphysis. Trap staining to observe the parameters of osteoclast surface per bone surface (Oc.S/BS) in region of the femur metaphysis to determine bone osteoclastic resorption.

\subsection{Hindlimbs blood flow measurements}

In the FAL group and the FAL\& MSCT group, the variation of blood flow in the hindlimbs of mice were observed using Laser Speckle Imaging System (Reward, China) on the day 1, 3, 7, 14 and 21 after FAL surgery. Anesthetize the mice by inhaling $2.0 \%$ isoflurane with a small animal anesthesia machine, and maintain with $1.0 \%$ isoflurane/oxygen under anesthesia. The hair on the abdomen and both hindlimbs were shaved, and the hindlimbs blood flow was scanned by fixing mice at supine position on the blood flow meter table. Both hindlimbs were imaged simultaneously with a pixel frame size of $993565 \mu \mathrm{m}^{2}$. Comparison of blood flow ratios (express as percent perfusion) in the ischemic (left) leg and intact (right) leg by analyzing digital color-coded images.

\subsection{Microfil-perfusion and CT-based microangiography}

The distribution of blood vessels within the femur in the FAL and FAL\& MSCT groups was assessed by perfusion of Microfil compound (MICROFIL, MV-122; Flow Tech, USA) on the day 1, day 14, and day 28 after FAL surgery. Mice were anesthetized with $10 \%$ chloral hydrate intraperitoneally and fixed on a foam table. An incision was formed between the glabella and abdomen to open the thoracic cavity. Before perfusion, an incision was made in the right atrial appendage and PBS (pH 7.4) and PBS containing heparin sodium (Solarbio, China) $(100 \mathrm{U} / \mathrm{ml}$ ) was flushed through the left ventricle at $1000 \mathrm{ml} / \mathrm{h}$. Then, 20 $\mathrm{ml}$ of Microfil compound was injected into the left ventricle at a rate of $3 \mathrm{ml} / \mathrm{min}$. After preserving the samples at $4^{\circ} \mathrm{C}$ for $24 \mathrm{~h}$, both hindlimb femurs were stripped from the hip joint and in $4 \%$ paraformaldehyde for $48 \mathrm{~h}$ of fixation. Then the femurs were decalcified in decalcification solution (Maxim, China) for 24h. Reconstructive scans were performed on the femurs of mice using micro-CT system, and the vessel volume relative to the tissue volume (VV/TV) was calculated.

\subsection{Immunofluorescence staining}


$4 \%$ paraformaldehyde fixation of the femur for $48 \mathrm{~h}$ and decalcified by shaking with $0.5 \mathrm{M}$ EDTA (Solarbio, China) for 24-48h. After decalcification, the femurs were incubated overnight with a $30 \%$ sucrose solution. The frozen femur embedded with the optimal cutting temperature compound (OCT) (Sakura, USA) was sliced into $10 \mu \mathrm{m}$-thick sections by using a cryostat (Leica, Germany). Tissue sections were air-dried in a cryostat and permeabilized in $0.3 \%$ Triton X- 100 (Solarbio, China) for $15 \mathrm{~min}$, blocked in $5 \%$ goat serum for $30 \mathrm{~min}$ at room temperature and incubate overnight with $5 \%$ goat serum primary antibody diluted in PBS at $4^{\circ} \mathrm{C}$. The following antibodies were used: CD31 (1:200, Abcam, USA), Endomucin (EMCN, 1:200, R\&D, USA). After incubation with the primary antibody, sections for 3 washes with PBS and incubated with fluorescently coupled secondary antibody (Alexa Fluor 488 and 594; Thermo Fisher, USA) in the dark at room temperature for 1 hour. Sections were then restained with 4',6-diamidino-2-phenylindole (DAPI), and the images are collected on a confocal microscope in Leica sp5.

\subsection{Statistical analysis}

GraphPad Prism software (version 9.0) was used for analysis and visualization of all data in this experiment. The data provided herein are expressed as the mean \pm standard deviation (SD) of the means from at least three experimental replicates. We used the Shapiro Wilkinson test to check that the data conformed to normality. Differences between groups were compared using the unpaired two-tailed $t$-test, and differences were considered statistically significant at $P<0.05$.

\section{Results}

\subsection{Differentiation capacities and characterization of mouse MSCs}

Primary MSCs were acquired from the femoral bone marrow of C57BL/ 6 mice. At $1-3$ days after starting to grow in culture flasks, most of the MSCs appeared as round or small bright cells of similar round size under an inverted microscope (Fig. 1A). After 4-7 days, the structure of MSCs appeared polygonal or spindly, and MSCs started stable passages (Fig. 1B-C). We induced osteogenic, lipogenic and chondrogenic differentiation of the MSCs. It was demonstrated that the obtained primary MSCs had stemness with multidirectional differentiation (Fig. 1D-F). Cell surface markers were also analyzed for MSCs using flow cytometry. The flow cytometry analysis indicated that the extracted cells highly expressed CD29, CD44, Sca-1 (Fig. 1G-I) and lowly expressed CD11b, CD45, CD117, (Fig. 1J-L). All these features conformed to the features of MSCs.

\subsection{MSCT prevented bone loss caused by OVX}

To evaluate the effects of MSCs on osteoporosis in vivo, sham and OVX group mice were treated with PBS, and OVX \& MSCT group mice were treated with $1 \times 10^{6}$ MSCs. The injection was performed by tail vein at day 7 and day 21 after OVX surgery. At day 28 post-surgery, the mice were sacrificed, and the femurs were collected from the sham group, OVX group and OVX \& MSCT group for scanning by microCT. The distal femoral trabeculae and mid-femoral cortical bone were reconstructed and analyzed 
(Fig. 2A). Our results indicated that Tb.BV/TV $(P=0.0022)$, Tb.N $(P=0.001)$ and Tb.BMD $(P=0.0002)$ in mice after receiving OVX were significantly decreased. Compared to the OVX group, the bone trabeculae in the OVX \& MSCT groups showed a significant increase in Tb.BV/TV $(P=0.0002), \mathrm{Tb} . \mathrm{N}(P<0.0001)$ and Tb.BMD $(P<0.0001)$ (Fig. 2B). The results of the analysis of $\mathrm{Ct}$ showed that Ct.Ar/Tt.Ar, Ct.Th and Ct.BMD were not statistically significant $(P>0.05$, Fig. $2 C$ ). These results indicated that MSCT significantly reduces the bone loss of $\mathrm{Tb}$ induced by denudation in mice.

\subsection{MSCT promotes bone formation and inhibits bone resorption in the distal femur}

We evaluated the morphological changes after MSCT by HE and Masson staining of bone tissue. HE staining results showed that no significant morphological changes were observed among groups (Fig. 3A). Moreover, Masson staining showed maturity of bone and collagen (Fig. 3B). Semi-quantitative analysis of the blue areas of Masson staining (Fig. 3D) showed that the new bone formation decreased in OVX group $(P<0.0001)$ and increased in OVX \& MSCT group $(P=0.0002)$. Trap staining of femur sections was performed to detect osteoclasts (purple-red) and calculate N.Oc/BS (Fig. 3C\&E). The size of the area of osteoclasts demonstrated that OVX could increase the bone resorption $(P<0.0001)$, but the process is inhibited by MSCT $(P<0.0001)$. These results demonstrate that MSCT is able to maintain the morphology and the amount of collagen fibers in Tb, as well as to inhibit bone resorption.

\subsection{MSCT facilitate revascularization in hindlimbs}

To elucidate the biological function of MSCs in restoring blood circulation, we performed FAL on the left hindlimb of the mice after OVX, and the opposite hindlimb was sham operated as the control (Fig. 4A). MSCs were subsequently transplanted for FAL\& MSCT groups at 24 hours and 14 days after FAL, and the FAL group received equal volumes $(200 \mu \mathrm{l})$ of PBS by tail vein injections at the corresponding time points. We performed a dynamic assessment of the overall hindlimb blood perfusion and flow rate using Laser Speckle Imaging System (Fig. 4B). Blood perfusion levels on the ligated side (left side) were similar in the FAL and FAL \& MSCT groups on day 1 . At day 7 to 14 , blood perfusion on the left side gradually recovered in the FAL\& MSCT group, whereas the recovery of blood perfusion was poorer in the FAL group. At day 21, the difference in left-sided blood perfusion between the two groups was significant, MSCT promoted blood reperfusion in the ischemic limb $(P<0.01$, Fig. 4C). However, on the right side, blood perfusion was not affected by MSCT $(P>0.05$, Fig. 4D).

To further clarify the effect of ligation of the femoral artery on the intraosseous vasculature, we evaluated the distribution of blood vessels within the hindlimb femurs at day 1, 14 and 28 after FAL by microfil perfusion (Fig. 4G). On the day 1 after FAL, the number of intraosseous vessels on the left side was significantly reduced in both two groups. With increasing time, the number of intraosseous vessels on the left side of the mice recovered, which was more rapidly in FAL\&MSCT group $(P<0.00001$, Fig. 4E). At day 28 , there was a significant variation in right-sided blood perfusion in the FAL \& MSCT group compared to the FAL group ( $P=0.028$, Fig. $4 \mathrm{~F})$. Taken together these results indicate that MSCT could promote the establishment of collateral circulation and intraosseous vascular remodeling at the ischemic site. 


\subsection{MSCT prevents bone loss in mice caused by reduced intraosseous blood perfusion}

We further evaluated whether the reduction in vascular perfusion had an effect on bone volume. By comparing the micro-CT results of the femur on the left side with the right side at day 28 after FAL in mice (Fig. 5A), we found whether or not they were injected with MSCs, Tb.BV/TV $(P=0.0003$, injection; $P=$ 0.0488, no injection), Tb.N $(P<0.0001$, injections; $P<0.0001$, no injection) and Tb.BMD $(P<0.0001$, injections; $P<0.0001$, no injection) on the left side compared with the right side were significantly reduced (Fig. 5B). In contrast, compared with the PBS injection, MSCT not only promoted microcirculation generation and somewhat avoided bone loss due to ligation $(P<0.0001)$. In addition, we did not observe an effect of the altered blood flow on Ct.Ar/Tt.Ar, Ct.Th and Ct.BMD $(P>0.05$, Fig. 5C). The results show that the decrease in vascular perfusion could accelerate the decline of cancellous bone mass, while MSCT could avoid bone loss due to the same reason.

\subsection{MSCT raised the number of H-type vessels in the femoral epiphysis}

Previous studies have shown that $\mathrm{H}$-type blood vessels in bone tissue are mainly found in the metaphysis and subperiosteum of long bones, which are important carriers of the coupling relationship between osteogenesis and angiogenesis. The reduction of $\mathrm{H}$-type blood vessels in bone occurs synergistically with the decrease of bone density, which is an early sensitive indicator of bone mass changes. Therefore, we performed immunofluorescence staining of bone tissue to investigate the alterations in the number of $\mathrm{H}$ type vessels in each group of this study (Fig. 6A). Integrated optic density (IOD) statistical analysis of CD31 and EMCN expression in the cancellous bone region of the epiphysis was performed in each group at day 28 (Fig. 6B-C). The results showed that OVX significantly reduced the expression of CD31 $(P=$ $0.0004)$ and EMCN $(P=0.0002)$. Ligation of the femoral artery further led to a decrease in CD31 $(P<$ $0.0001)$ and $\mathrm{EMCN}(P<0.0001)$ expression. The expression of CD31 $(P=0.015, \mathrm{OVX} ; P=0.0004, \mathrm{FAL})$ and $\mathrm{EMCN}(P=0.0049, \mathrm{OVX} ; P=0.0002, \mathrm{FAL})$ was effectively increased by MSCT after either OVX or FAL procedures. By counting the number of H-vessels per unit area (Fig. 6D), MSCT was shown to promote an increase in the number of $\mathrm{H}$-type vessels after $\operatorname{OVX}(P=0.0162)$ and $\mathrm{FAL}(P=0.0042)$. These results were consistent with the intraosseous vascular perfusion, indicating that MSCT plays a positive role in intraosseous vascular remodeling and improvement of local microcirculation.

\section{Discussion}

Postmenopausal osteoporosis is one of the most common types of primary osteoporosis, which is characterized by decreased ovarian function and low estrogen levels, resulting in decreased BMD and destruction of bone structure. ${ }^{[20 ; 21]}$. Individualized treatment for osteoporosis patients requires selection reasonable therapeutic options according to patient's specific situation ${ }^{[22-24]}$. Osteoporosis prevention and treatment is indispensable to avoid osteoporotic fractures. MSCT is a new potential therapeutic method for postmenopausal osteoporosis and has been demonstrated to enhance bone mass in mice 
[25]. In this study, we focused on the role of MSCs in treating bone mass and altered microcirculation within the bone in ovariectomized mice. These results open up new horizons for the potential clinical application of MSCT in the treatment of postmenopausal osteoporosis.

The formation of osteoporosis is accompanied by the destruction of blood vessels in the bone, and the two factors may also be mutually promoting ${ }^{[26]}$. Bone is a sufficiently vascularized tissue that has a substantial network of blood vessels, and the blood content in bone can be 10-15\% of the cardiac output at rest ${ }^{[27]}$. The vascular network structure in bone not only plays a role in providing nutrients and transporting metabolic wastes, but is also essential for the stability, development and repair of bone

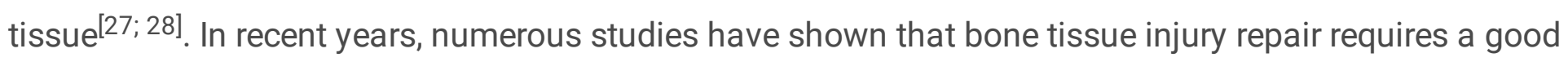
blood supply. The richer the bone blood flow, the stronger the bone tissue repair capacity ${ }^{[29-31]}$. The incidence of osteoporosis can be attributed to poor microcirculation within the bone, leading to such factors as increased oxidative stress, inflammation, and nutrient deficiencies ${ }^{[32 ; 33]}$. In contrast, such as estrogen, calcitonin, and bisphosphonates all have positive effects on the maintenance of intraosseous vascularity ${ }^{[34-36]}$. Therefore, exploring more therapeutic strategies that can improve intraosseous microcirculation to treat of osteoporosis are crucial for clinical applications ${ }^{[37]}$. This experiment verified that the bone repair of MSCs in the animal model of osteoporosis is related to the role of promoting blood vessels. After the infusion of MSCs, it is more obvious in preventing the bone loss of castrated mice and promoting the recovery of hindlimb blood vessels.

MSCs are the most commonly used cell source for bone tissue engineering, and they have a variety of differentiation potentials such as osteogenic, chondrogenic and vascular. Therefore, MSCs are widely used to bone repair and bone regeneration $\left.{ }^{[38 ;} 39\right]$.The pathological mechanism of osteoporosis may be related to the decline in the differentiation ability and the number of MSCs in vivo ${ }^{[40]}$. Therefore, injection of MSCs can effectively supplement the number of MSCs in the body and promote bone formation to treat osteoporosis. There were also studies that proposed that the therapeutic effects of MSCs are mainly attributed to paracrine mechanisms ${ }^{[41 ; 42]}$. A large number of soluble factors produced by paracrine action are involved in angiogenic, anti-apoptotic, anti-inflammatory and immunomodulatory processes ${ }^{[43-46]}$. These soluble factors can improve the microenvironment, facilitate neoangiogenesis and remodel the damaged tissues ${ }^{[47]}$. In this study, MSCs were used to treat estrogen deficiency-induced osteoporosis, and we found that Tb.BV/TV, Tb.N, and Tb.BMD in ovariectomized mice were increased after MSCT. Meanwhile, the osteoclasts in in the metaphysis was significantly decreased after MSCs infusion treatment. These findings reveal a significant inhibitory effect of MSCs on bone resorption and a significant efficacy in preventing osteoporosis caused by estrogen reduction.

MSCs have a significant effect on improving microcirculation after infusion ${ }^{[48]}$. Studies have reported that MSCs have a positive effect on alleviating the symptoms of lower limb ischemia ${ }^{[49]}$, promoting wound angiogenesis ${ }^{[50]}$, and restoring cerebral ischemia ${ }^{[51]}$. However, its role in promoting intra-osseous vascular remodeling is not very clear ${ }^{[52]}$. In this study, after constructing an animal model of 
ovariectomized mice, it was observed that the decrease in bone mass in mice was associated with a reduction in the number of blood vessels in the bone. In addition, we used an animal model of limb ischemia caused by the closure of a completely ischemic femoral artery and its branches. After ligating the femoral artery of the lower extremity of the mouse, it was observed that the blood flow in the bone was reduced, which led to the reduction of bone mass. The tail vein injection of BMSCs can help the ischemic limb re-establish blood supply, it also includes the restoration of blood circulation in the bone and raising the number of $\mathrm{H}$-vessels in the epiphysis. Analysis of bone mass in ischemic parts of lower limbs found that MSCT can effectively prevent bone mass loss caused by reduced blood flow. In the OVX and FAL models, we only observed the changes in bone mass of mouse femurs within 5 weeks. There were no significant changes observed in bone mass in cortical bone in this study, and MSCT did not have a significant impact on it. This phenomenon is consistent with the pattern of osteoporosis, which begins

with bone loss of cancellous bone in early osteoporosis, followed by thinning of cortical bone ${ }^{[53 ; 54]}$. This result we speculate may be related to the poorer sensitivity of cortical bone to ischemia compared to trabeculae, but more research is needed evidence to support this view.

As the research progresses, the role of blood vessels in tissue repair has been re-understood. Since the characteristics of MSCs in regeneration and better angiogenesis potential given due attention, MSCs becoming effective therapy for osteoporosis. Therefore, when treating osteoporosis, not only need to consider increasing bone mass, but also need to consider vascular repair, which also provides a better guarantee for ensuring the treatment effect of osteoporosis.

\section{Conclusion}

This study provides evidence for the positive role of MSCs in participating in endosteal vascular remodeling and reducing bone loss after tail vein injection. We observed that the reduction of intraosseous vascularity leads to the development of osteoporosis, and MSCT is closely associated with the re-establishment of intraosseous vascularity and the prevention of bone loss. The results of this study may serve as one of the reasons to explain the effectiveness of MSCs in the treatment of osteoporosis. In conclusion, this study reveals the role of MSCs in promoting endosteal revascularization to reduce bone loss, and provides a theoretical reference and practical basis for MSCs in the treatment of postmenopausal osteoporosis.

\section{Abbreviations}




\begin{tabular}{ll} 
MSCs & Mesenchymal stem cells \\
\hline MSCT & MSCs transplantation \\
\hline DMEM & Dulbecco's modified eagle medium \\
\hline FBS & Fatal bovine serum \\
\hline PBS & Phosphate buffer saline \\
\hline OVX & Ovariectomy \\
\hline FAL & Femoral artery ligation \\
\hline ROI & Region of interest \\
\hline BV/TV & Bone volume per tissue volume \\
\hline N & Number \\
\hline BMD & Bone mineral density \\
\hline Tb & Trabecular \\
\hline Th & Thickness \\
\hline Ar/Tt.Ar & Ratio of area to total area \\
\hline Ct & Cortical bone \\
\hline EDTA & Ethylene diamine tetraacetic acid \\
\hline CF/BS & Collagen fiber per bone surface \\
\hline Oc.S/BS & Osteoclast surface per bone surface \\
\hline WV/TV & Vessel volume relative to the tissue volume \\
\hline OCT & Optimal cutting temperature compound \\
\hline EMCN & Endomucin \\
\hline DAPI & 4 ',6-diamidino-2-phenylindole \\
\hline SD & Standard deviation \\
\hline IOD & Integrated optic density \\
\hline
\end{tabular}

\section{Declarations}

Authors' contributions

W.WZ., W.YH., T.ZH. conceived and designed the study. W.WZ., W.YH. contributed to wrote of the paper. W.WZ., W.YH., T.ZH., C.YC., L.Z. performed the experiments and collected and analyzed the data D.H., Z.ZY. assisted in data collection, extraction, and evaluation of the eligibility of the original data. H.F. 
provided technical support for the analysis and critical revision of the manuscript. All authors reviewed and approved the final manuscript.

\section{Funding}

This study was supported by Yunnan Province Major Science and Technology Special Projects (2019ZF002), Outstanding Medical Academic Leader Project under grant From Yunnan (L-201621), Industrial Technology Leader Program from Yunnan (YLXL20170046), Yunnan Provincial Education Department Scientific Research Fund (2020Y0123), Academician Workstation of Qiu Yong in Yunnan Province (202005F150038), Yunnan Province Clinical Center for Bone and joint Diseases (ZX2019-03-04).

\section{Availability of data and materials}

Not applicable.

\section{Ethics approval and consent to participate}

Mouse were used under the ethical approval and the ethical guidelines of the Ethics Committee of Kunming Medical University.

\section{Consent for publication}

Not applicable.

\section{Competing interests}

The authors declare no conflict of interest.

\section{Footnotes}

Publisher's Note

Springer Nature remains neutral with regard to jurisdictional claims in published maps and institutional affiliations.

\section{References}

1. Shams-White MM, Chung M, Du M, et al. Dietary protein and bone health: a systematic review and meta-analysis from the National Osteoporosis Foundation. Am J Clin Nutr. 2017;105(6):1528-43.

2. Yang TL, Shen $\mathrm{H}$, Liu A, et al. A road map for understanding molecular and genetic determinants of osteoporosis. Nat Rev Endocrinol. 2020;16(2):91-103.

3. Walker-Bone K, Walter G, Cooper C. Recent developments in the epidemiology of osteoporosis. Curr Opin Rheumatol. 2002;14(4):411-5. 
4. Jiang $Y$, Zhang $P$, Zhang $X$, et al. Advances in mesenchymal stem cell transplantation for the treatment of osteoporosis. Cell Prolif. 2021;54(1):e12956.

5. Anastasilakis AD, Polyzos SA, Yavropoulou MP, et al. Combination and sequential treatment in women with postmenopausal osteoporosis. Expert Opin Pharmacother. 2020;21(4):477-90.

6. Gatti D, Fassio A. Pharmacological management of osteoporosis in postmenopausal women: The current state of the art. J Popul Ther Clin Pharmacol. 2019;26(4):e1-17.

7. Zhou S, Huang G, Chen G. Synthesis and biological activities of drugs for the treatment of osteoporosis. Eur J Med Chem. 2020;197:112313.

8. Gallagher JC. Advances in osteoporosis from 1970 to 2018. Menopause. 2018;25(12):1403-17.

9. Armas LA, Recker RR. Pathophysiology of osteoporosis: new mechanistic insights. Endocrinol Metab Clin North Am. 2012;41(3):475-86.

10. Zhao W, Shen G, Ren H, et al. Therapeutic potential of microRNAs in osteoporosis function by regulating the biology of cells relate $d$ to bone homeostasis. J Cell Physiol. 2018;233(12):9191-208.

11. Black DM, Rosen CJ. Clinical Practice. Postmenopausal Osteoporosis. N Engl J Med. 2016;374(3):254-62.

12. Ding $\mathrm{W}, \mathrm{Xu} \mathrm{C}$, Zhang $\mathrm{Y}$, et al. Advances in the understanding of the role of type-H vessels in the pathogenesis of osteoporosis. Arch Osteoporos. 2020;15(1):5.

13. Shi L, Huang H, Lu X, et al. Effect of human umbilical cord-derived mesenchymal stem cells on lung damage in severe COVID-19 patie nts: a randomized, double-blind, placebo-controlled phase 2 trial. Signal Transduct Target Ther. 2021;6(1):58.

14. Krze?Niak AM, Radzimowski K, Stolarczyk A. Comparison of the treatment results of knee osteoarthritis using adipose tissue mesenchymal stromal c ells derived through enzymatic digestion and mechanically fragmented adipose tissue. Med (Baltim). 2021;100(9):e24777.

15. Kiernan J, Davies JE, Stanford WL. Concise Review: Musculoskeletal Stem Cells to Treat Age-Related Osteoporosis. Stem Cells TransI Med. 2017;6(10):1930-9.

16. Liu CB, Huang H, Sun P, et al. Human Umbilical Cord-Derived Mesenchymal Stromal Cells Improve Left Ventricular Function, Perfusion, and Remodeling in a Porcine Model of Chronic Myocardial Ischemia. Stem Cells TransI Med. 2016;5(8):1004-13.

17. Suda S, Nito C, Yokobori S, et al. Recent Advances in Cell-Based Therapies for Ischemic Stroke. Int J Mol Sci, 2020, 21(18).

18. Osipova OS, Saaia SB, Karpenko AA, et al. [Problems and prospects of cell therapy for critical ischaemia of lower limbs]. Angiol Sosud Khir. 2020;26(2):23-33.

19. Zhong G, Yao J, Huang X, et al. Injectable ECM hydrogel for delivery of BMSCs enabled full-thickness meniscus repair in an orthotopic rat model. Bioact Mater. 2020;5(4):871-9.

20. Gibon E, Lu L, Goodman SB. Aging, inflammation, stem cells, and bone healing. Stem Cell Res Ther. 2016;7:44. 
21. Infante A, Rodr"a guez Cl. Osteogenesis and aging: lessons from mesenchymal stem cells. Stem Cell Res Ther. 2018;9(1):244.

22. Rozenberg S, Al-Daghri N, Aubertin-Leheudre M, et al. Is there a role for menopausal hormone therapy in the management of postmenopausal osteoporosis? Osteoporos Int. 2020;31(12):2271-86.

23. Kataoka Y, Luo Y, Chaimani A, et al. Cumulative network meta-analyses, practice guidelines, and actual prescriptions for postmenopausal os teoporosis: a meta-epidemiological study. Arch Osteoporos. 2020;15(1):21.

24. Rees M, Angioli R, Coleman RL, et al. European Menopause and Andropause Society (EMAS) and International Gynecologic Cancer Society (IGCS) position statement on managing the menopause after gynecological cancer: focus on menopausal symptom $s$ and osteoporosis. Maturitas. 2020;134:56-61.

25. Aghebati-Maleki L, Dolati S, Zandi R, et al. Prospect of mesenchymal stem cells in therapy of osteoporosis: A review. J Cell Physiol. 2019;234(6):8570-8.

26. Ramasamy SK, Kusumbe AP, Schiller M, et al. Blood flow controls bone vascular function and osteogenesis. Nat Commun. 2016;7:13601.

27. Tomlinson RE, Silva MJ. Skeletal Blood Flow in Bone Repair and Maintenance. Bone Res. 2013;1(4):311-22.

28. Brandi ML, Collin-Osdoby P. Vascular biology and the skeleton. J Bone Miner Res. 2006;21(2):18392.

29. Hu K, Olsen BR. The roles of vascular endothelial growth factor in bone repair and regeneration. Bone. 2016;91:30-8.

30. Sivaraj KK, Adams RH. Blood vessel formation and function in bone. Development. 2016;143(15):2706-15.

31. Jiang $X$, Xu C, Shi H, et al. PTH1-34 improves bone healing by promoting angiogenesis and facilitating MSCs migration and different iation in a stabilized fracture mouse model. PLoS One. 2019;14(12):e0226163.

32. Von Bahr L, Batsis I, Moll G, et al. Analysis of tissues following mesenchymal stromal cell therapy in humans indicates limited long-term engraftment and no ectopic tissue formation. Stem Cells. 2012;30(7):1575-8.

33. Lee K, Kim H, Kim JM, et al. Systemic transplantation of human adipose-derived stem cells stimulates bone repair by promoting oste oblast and osteoclast function. J Cell Mol Med. 2011;15(10):2082-94.

34. Lobo RA, Pickar JH, Stevenson JC, et al. Back to the future: Hormone replacement therapy as part of a prevention strategy for women at the ons et of menopause. Atherosclerosis. 2016;254:282-90.

35. Nakagami H, Osako MK, Shimizu H, et al. [Common molecular mechanism of osteoporosis and vascular diseases]. Nihon Ronen Igakkai Zasshi. 2011;48(2):146-8. 
36. Schoutens A, Verhas M, Dourov N, et al. Bone loss and bone blood flow in paraplegic rats treated with calcitonin, diphosphonate, and indometh acin. Calcif Tissue Int. 1988;42(2):136-43.

37. Szab“ ${ }^{\circledR}$ A, Janovszky, $P$ " ${ }^{\circledR} C s$, et al. The periosteal microcirculation in health and disease: An update on clinical significance. Microvasc Res. 2017;110:5-13.

38. Chen X, Wang J, Yu L, et al. Effect of Concentrated Growth Factor (CGF) on the Promotion of Osteogenesis in Bone Marrow Stromal Ce lls (BMSC) in vivo. Sci Rep. 2018;8(1):5876.

39. Wang X, Wang C, Gou W, et al. The optimal time to inject bone mesenchymal stem cells for fracture healing in a murine model. Stem Cell Res Ther. 2018;9(1):272.

40. Zhao F, Ma X, Qiu W, et al. Mesenchymal MACF1 Facilitates SMAD7 Nuclear Translocation to Drive Bone Formation. Cells, 2020, 9(3).

41. Li J, Zhang Q, Wang W, et al. Mesenchymal stem cell therapy for ischemic stroke: A look into treatment mechanism and therapeutic po tential. J Neurol, 2020.

42. Hutchings $G$, Janowicz $K$, Moncrieff $L$, et al. The Proliferation and Differentiation of Adipose-Derived Stem Cells in Neovascularization and Angioge nesis. Int J Mol Sci, 2020, 21(11).

43. Qin Y, Sun R, Wu C, et al. Exosome: A Novel Approach to Stimulate Bone Regeneration through Regulation of Osteogenesis and Angio genesis. Int J Mol Sci, 2016, 17(5).

44. Li Y, Jin D, Xie W, et al. Mesenchymal Stem Cells-Derived Exosomes: A Possible Therapeutic Strategy for Osteoporosis. Curr Stem Cell Res Ther. 2018;13(5):362-8.

45. Van De Vyver M, Powrie YSL, Smith C. Targeting Stem Cells in Chronic Inflammatory Diseases. Adv Exp Med Biol. 2021;1286:163-81.

46. Ueno Y, Hira K, Miyamoto N, et al. Pleiotropic Effects of Exosomes as a Therapy for Stroke Recovery. Int J Mol Sci, 2020, 21(18).

47. Alagiakrishnan K, Juby A, Hanley D, et al. Role of vascular factors in osteoporosis. J Gerontol A Biol Sci Med Sci. 2003;58(4):362-6.

48. Tang $\mathrm{H}, \mathrm{He} \mathrm{Y}$, Li L, et al. Exosomal MMP2 derived from mature osteoblasts promotes angiogenesis of endothelial cells via VEGF/Erk 1/2 signaling pathway. Exp Cell Res. 2019;383(2):111541.

49. Wang Z, Zheng L, Lian C, et al. Human Umbilical Cord-Derived Mesenchymal Stem Cells Relieve Hind Limb Ischemia by Promoting Angiogene sis in Mice. Stem Cells Dev. 2019;28(20):1384-97.

50. He Y, Xia J, Chen $\mathrm{H}$, et al. Human adipose liquid extract induces angiogenesis and adipogenesis: a novel cell-free therapeutic age nt. Stem Cell Res Ther. 2019;10(1):252.

51. Li L, Chu L, Ren C, et al. Enhanced Migration of Bone Marrow-Derived Mesenchymal Stem Cells with Tetramethylpyrazine and Its Syn ergistic Effect on Angiogenesis and Neurogenesis After Cerebral Ischemia in Rats. Stem Cells Dev. 2019;28(13):871-81.

52. Diomede F, Marconi GD, Fonticoli L, et al. Functional Relationship between Osteogenesis and Angiogenesis in Tissue Regeneration. Int J Mol Sci, 2020, 21(9).

53. Ramchand SK, Seeman E. The Influence of Cortical Porosity on the Strength of Bone During Growth and Advancing Age. Curr Osteoporos Rep. 2018;16(5):561-72. 
54. Samelson EJ, Broe KE, Xu H, et al. Cortical and trabecular bone microarchitecture as an independent predictor of incident fracture risk in older women and men in the Bone Microarchitecture International Consortium (BoMIC): a prospective study. Lancet Diabetes Endocrinol. 2019;7(1):34-43.

\section{Figures}
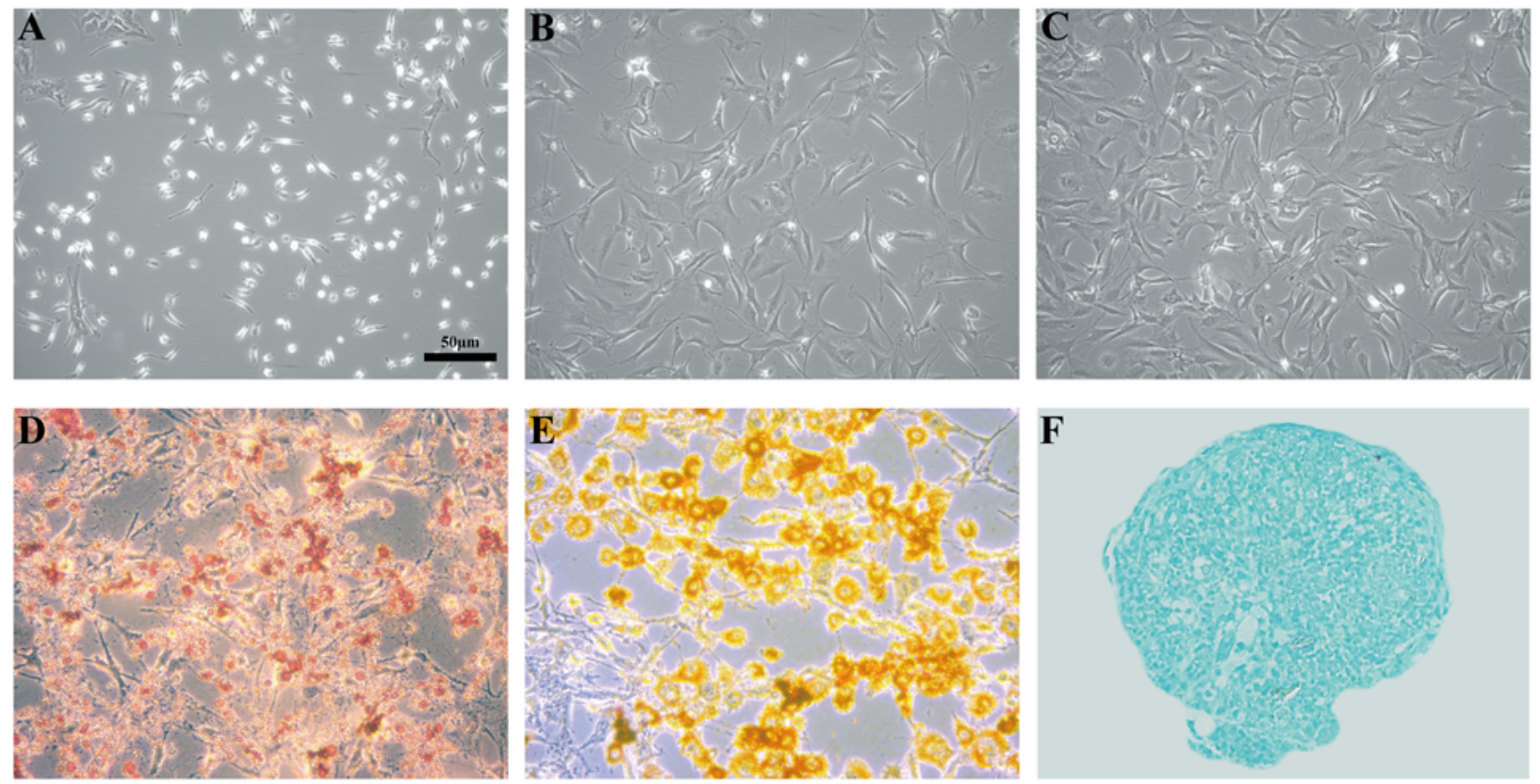

G

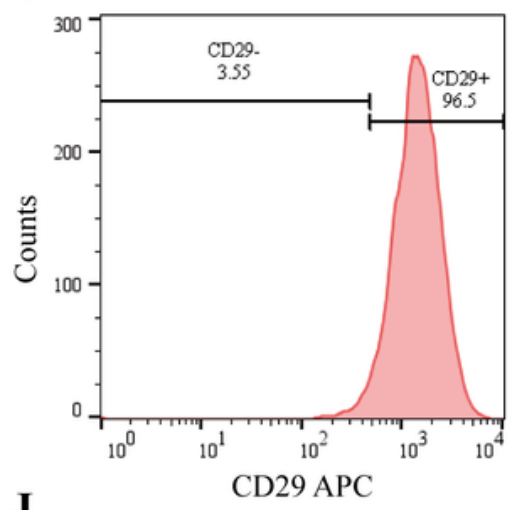

$\mathbf{J}$

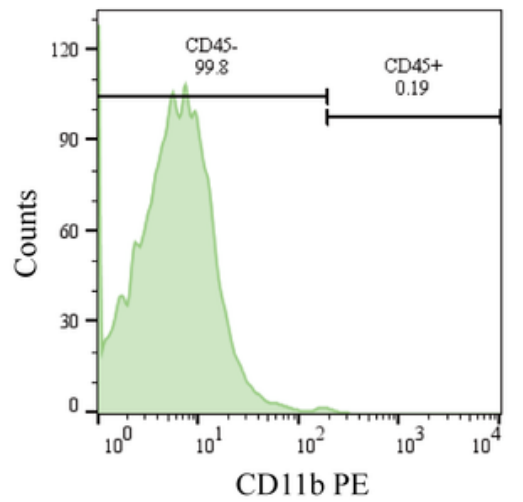

$\mathbf{H}$
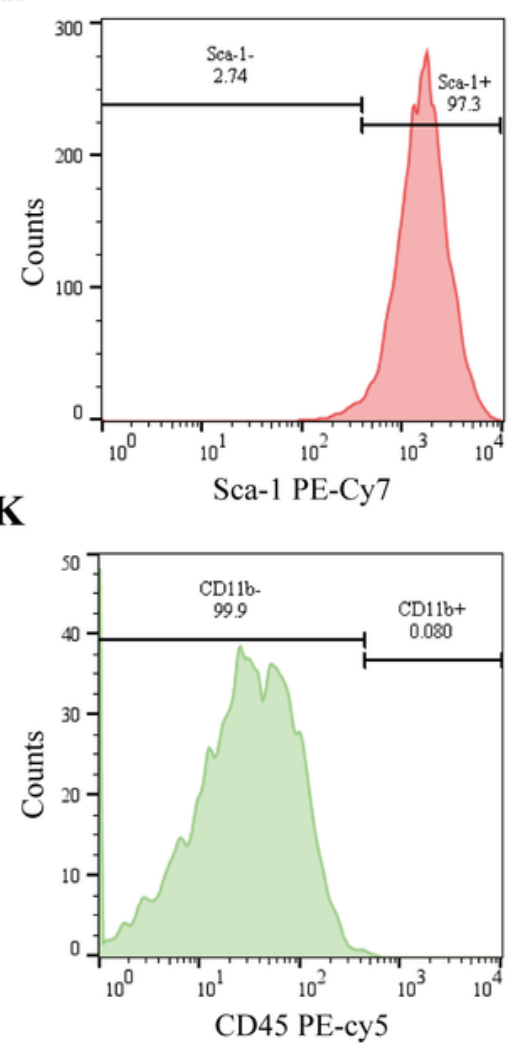

I
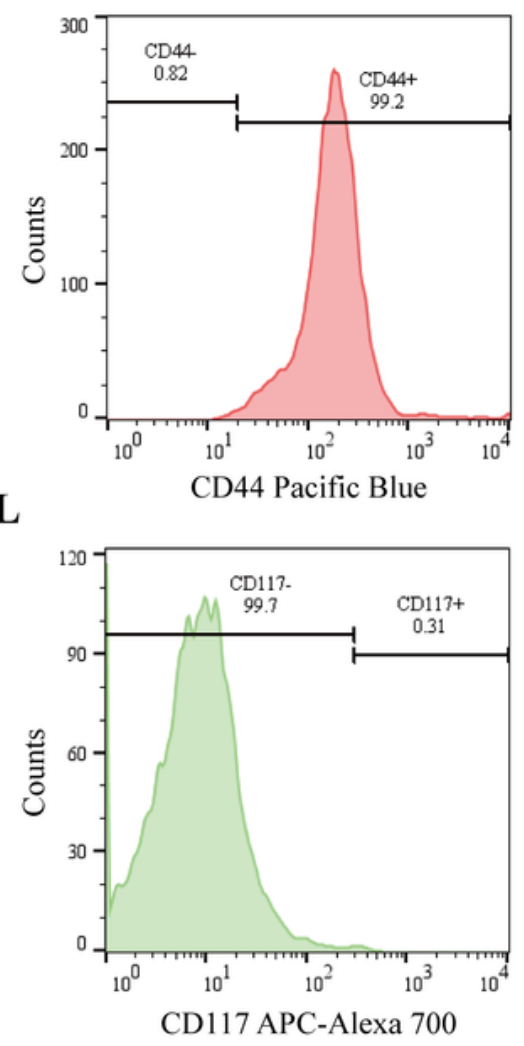
Culture and phenotypic identification of MSCs. Morphology of primary MSCs from C57BL/6 mice at (a-c) day 1, 4 and 7 under inverted microscope. Staining results of MSCs for (d) osteogenic induction, (e)

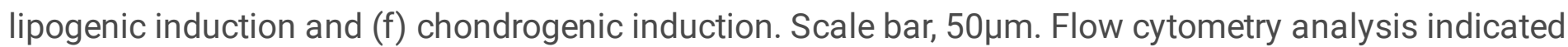
primary mouse MSCs were positive for (g) CD29, (h) Sca-1 and (i) CD44 and negative for (j) CD11b, (k) CD45 and (I) CD117.

A
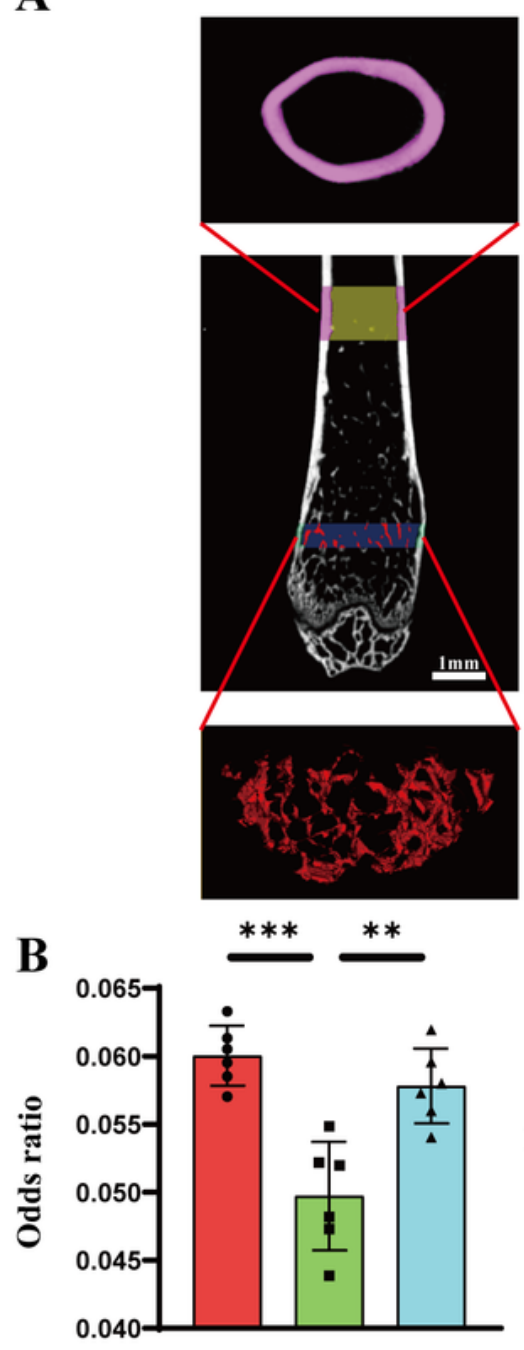

Tb.BV/TV

C

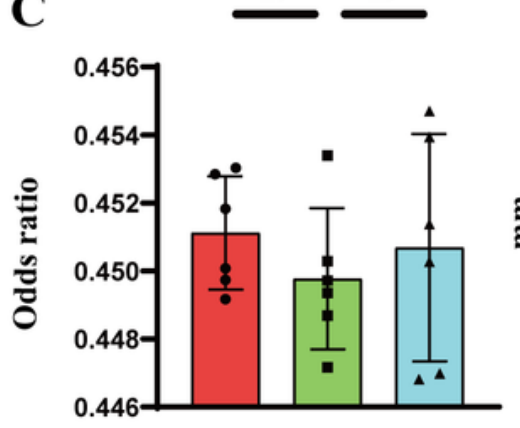

Ct.Ar/Tt.Ar
OVX
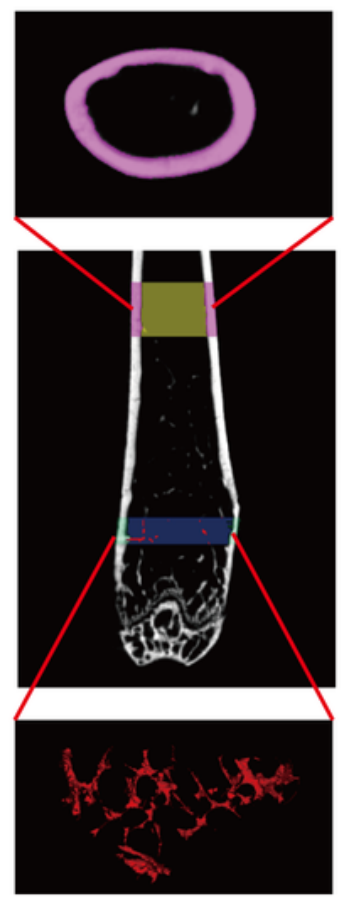

$* * * * \quad * * *$

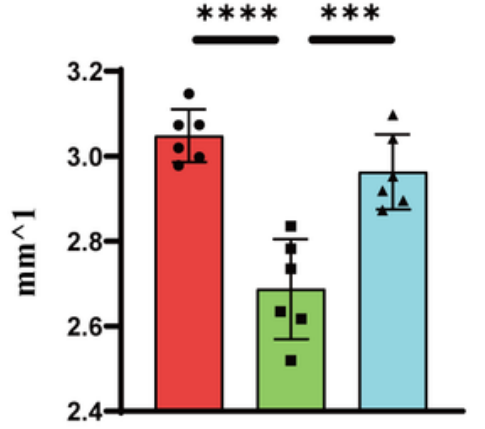

Tb.N

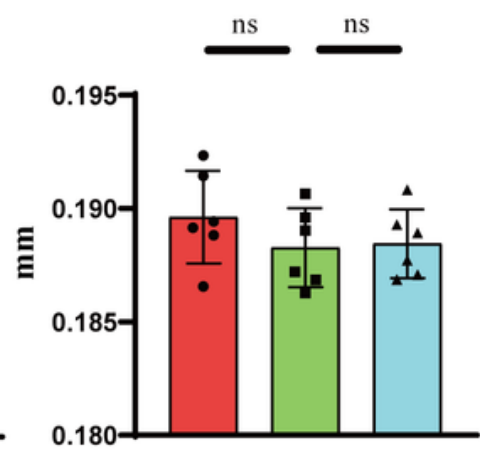

Ct.Th
OVX+MSCT
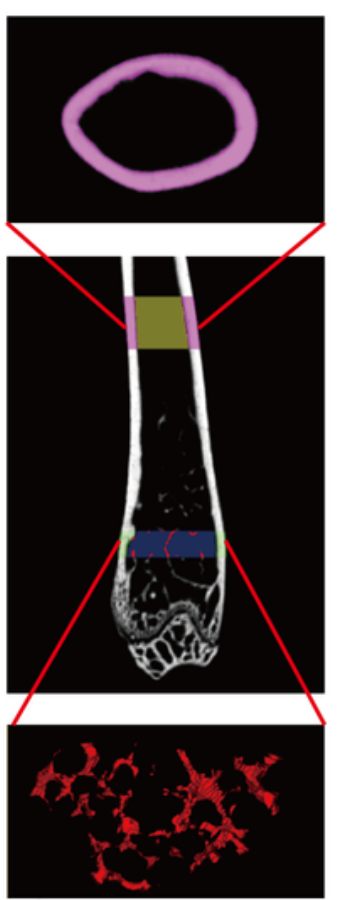

$* * * * \quad * * *$

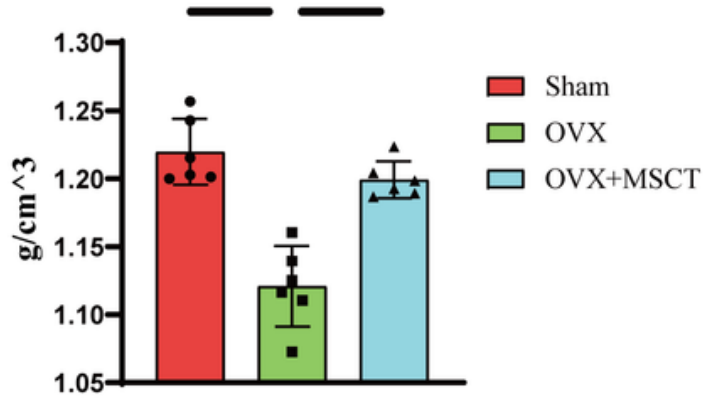

Tb.BMD

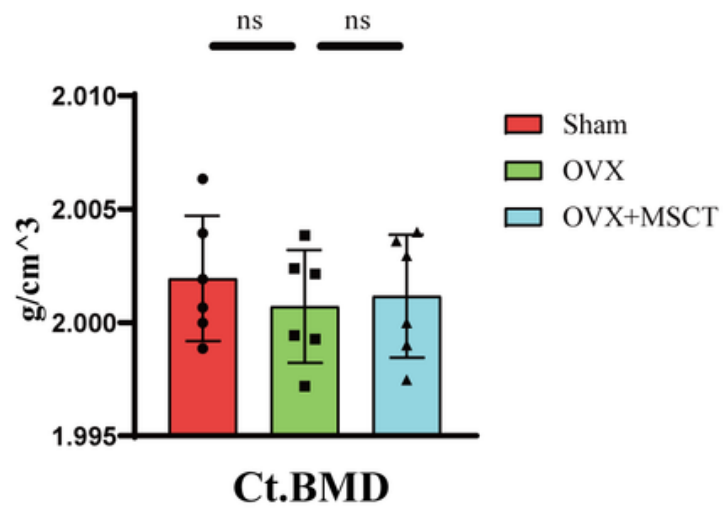

Figure 2 
Prevention of osteoporosis in ovariectomy-induced mice. (a) Micro-CT bone scan and CT-based threedimensional microangiography of mouse femur on day 35 after OVX surgery. (b) Quantitative analysis of Tb.BV/TV, Tb.N, and Tb.BMD. (c) Quantitative analysis of Ct.BMD, Ct.Th, and Ct.Ar/Tt.Ar. $\mathrm{n}=6$ per group. ${ }^{*} \mathrm{P}<0.05,{ }^{* * \mathrm{P}}<0.01, * * * \mathrm{P}<0.001$, ${ }^{* * *} \mathrm{P}<0.0001, \mathrm{~ns} \geq 0.05$.
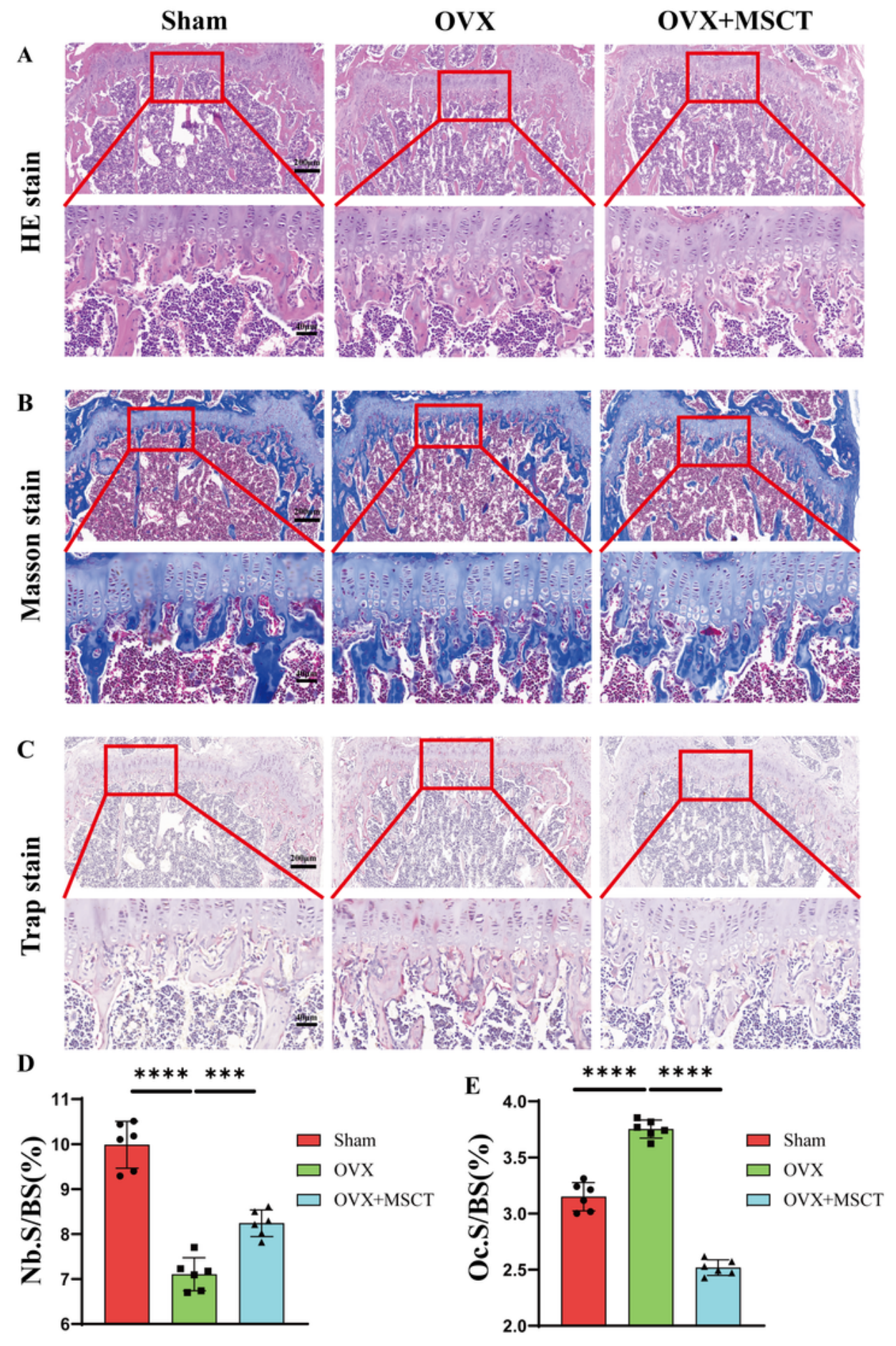

Figure 3 
Cell transplantation stimulates new osteogenesis and inhibits the number of osteoclasts. (a) HE staining results of mouse femur. (b) Masson staining results of mouse femur and (d) statistical analysis of Nb.S/BS. (c) Trap staining results of mouse femur and (e) the results of statistical analysis on Oc.S/BS. Scale bar, $200 \mu \mathrm{m}$ and $40 \mu \mathrm{m} . \mathrm{n}=6$ per group. ${ }^{\star} \mathrm{P}<0.05$, ${ }^{\star \star P}<0.01,{ }^{\star \star *} \mathrm{P}<0.001,{ }^{\star \star \star \star *} \mathrm{P}<0.0001, \mathrm{~ns} \geq$ 0.05 .

A

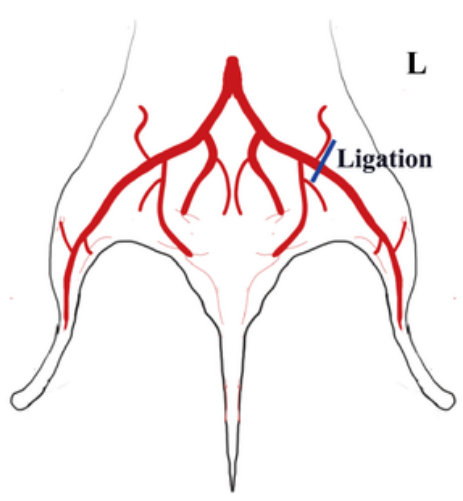

C

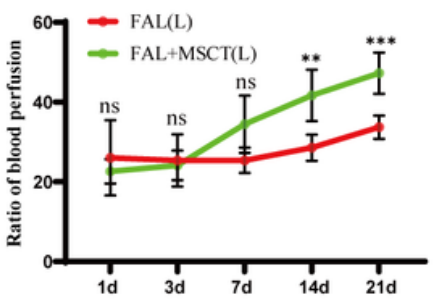

G

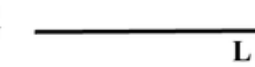

$\Xi$
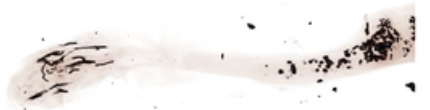

D

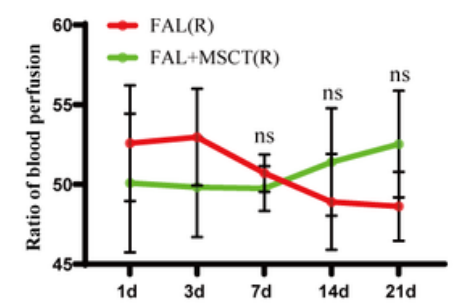

FAL+MSCT
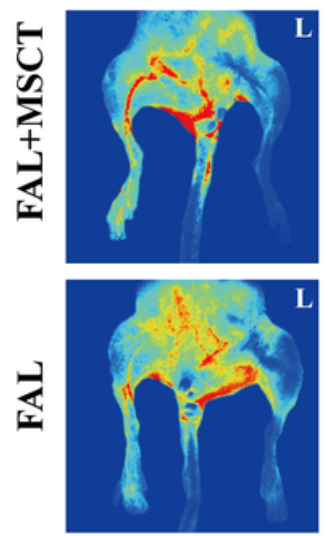

$\mathbf{E}$
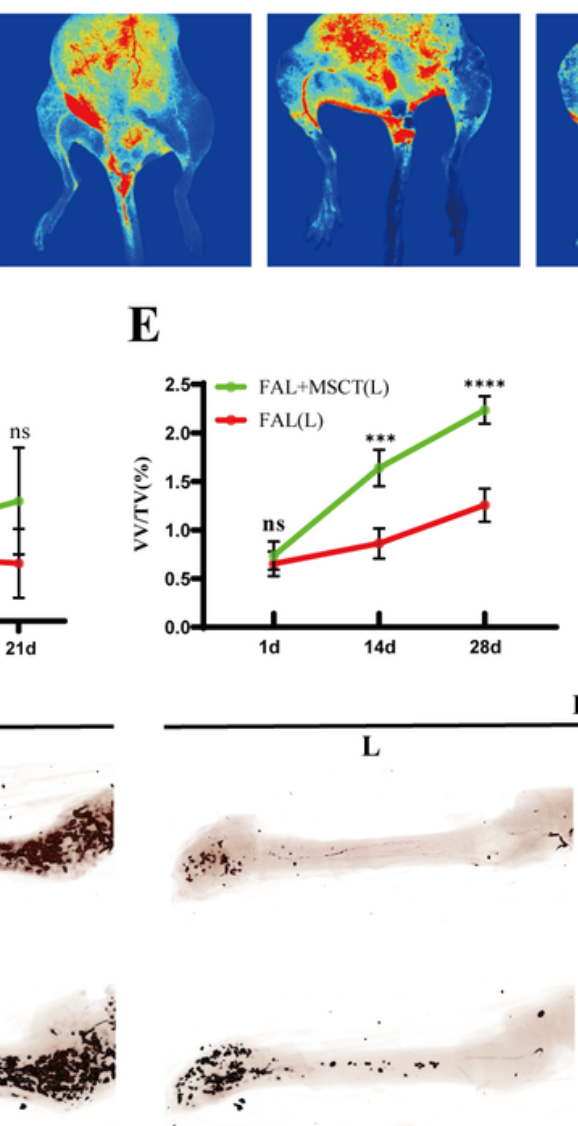

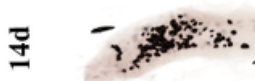
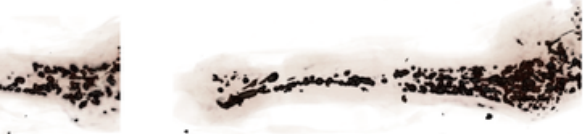

- Hysts s
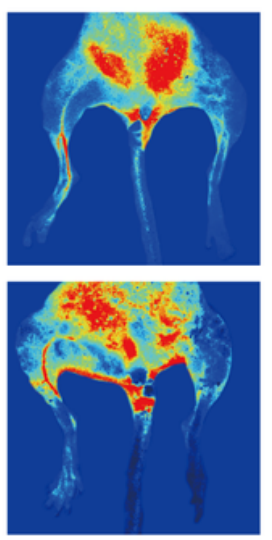

L
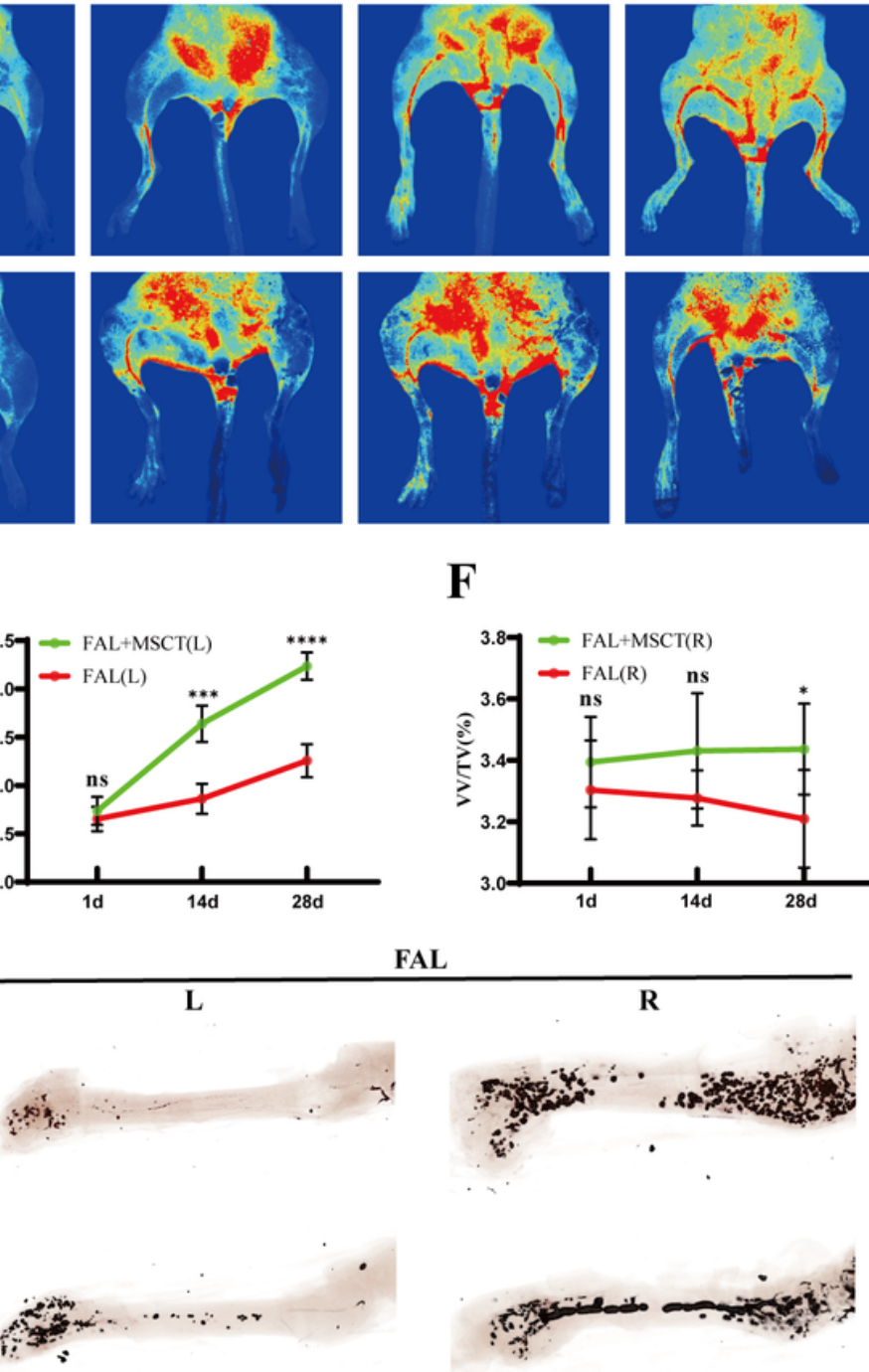

$\mathbf{F}$
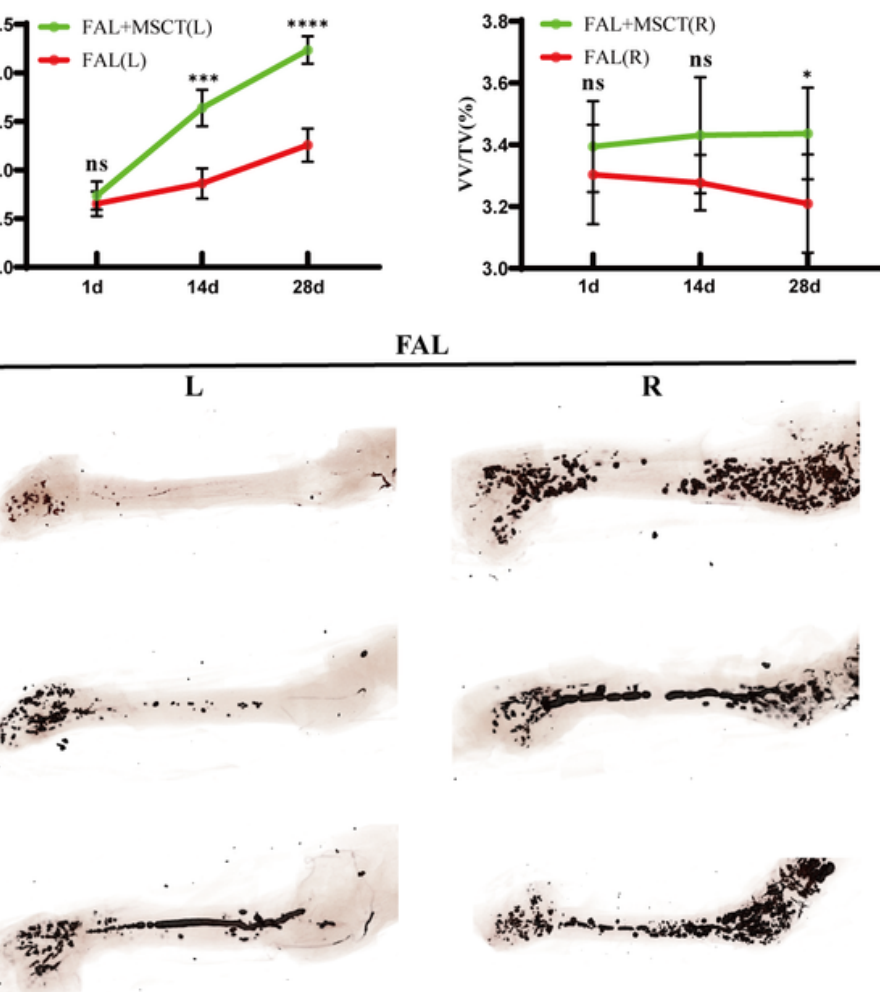

14d

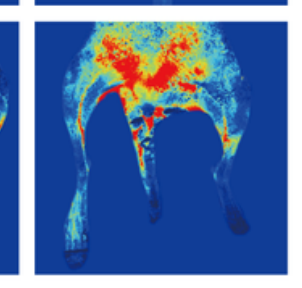

21d

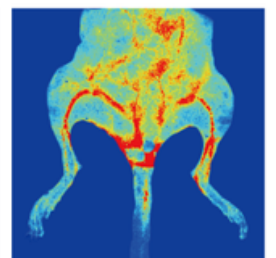

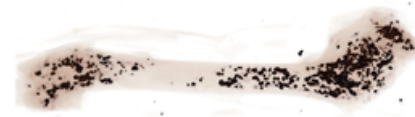

$\rightarrow$ -

\section{Figure 4}

Cell transplantation promotes microcirculation generation in the hindlimb of ligated arteries. (a) Diagram of femoral artery ligation in mice. (b) the blood flow changes with the increase of the Post-Operation days by Laser Speckle flowmetry (high perfusion red, no perfusion dark blue) and (c, d) quantitative analysis of blood flow (R: right side, L: left side). Three-dimensional reconstruction of $(\mathrm{g})$ vascular perfusion in mice 
and $(\mathrm{e}, \mathrm{f})$ quantitative analysis of vascular volumes. $\mathrm{n}=6$ per group. ${ }^{\star} \mathrm{P}<0.05,{ }^{*} \mathrm{P}<0.01,{ }^{*} \mathrm{P}<0.001$, $\star \star * * \mathrm{P}<0.0001, \mathrm{~ns} \geq 0.05$.

A
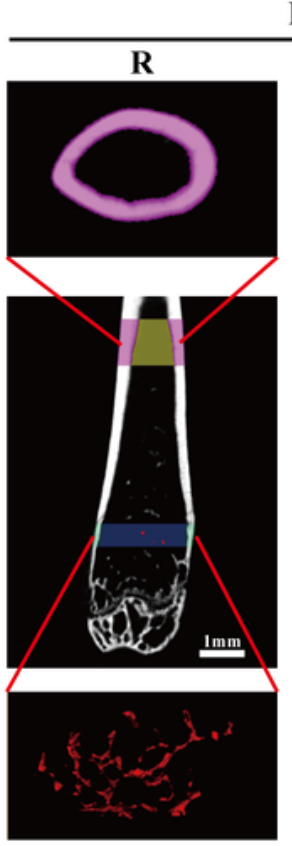

$\mathbf{B}$

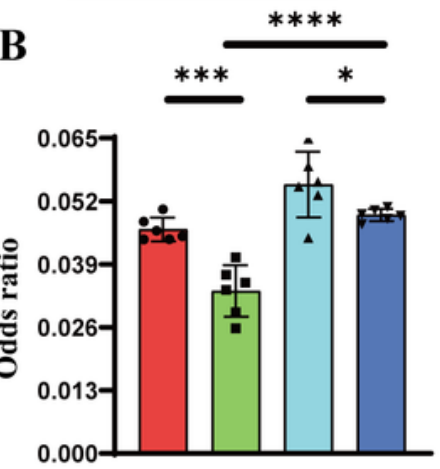

Tb.BV/TV

C

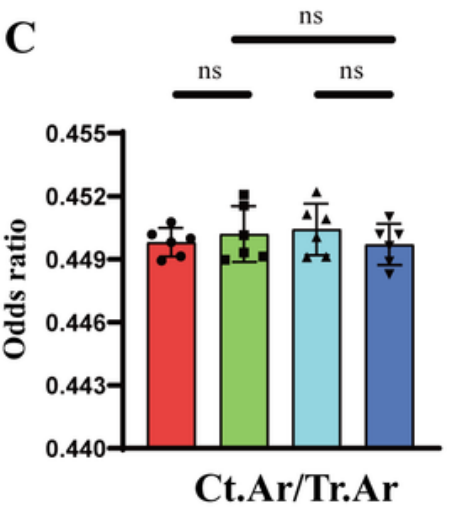

FAL
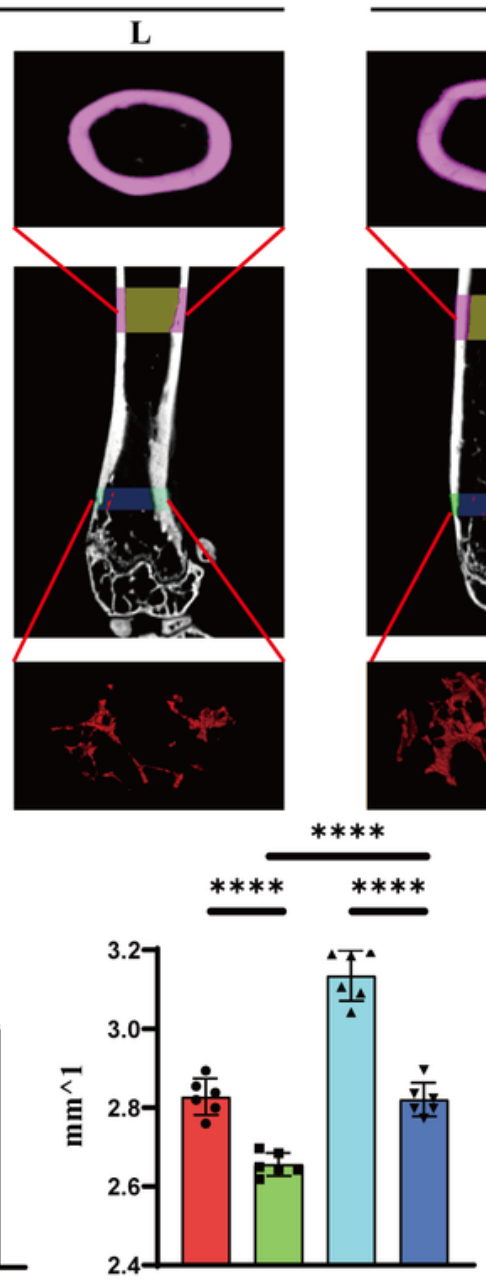

Tb.N

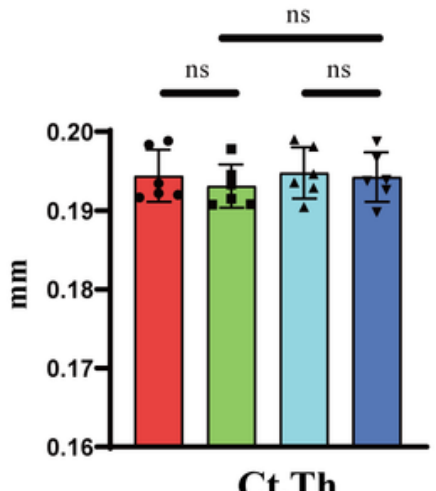

FAL+MSCT

R
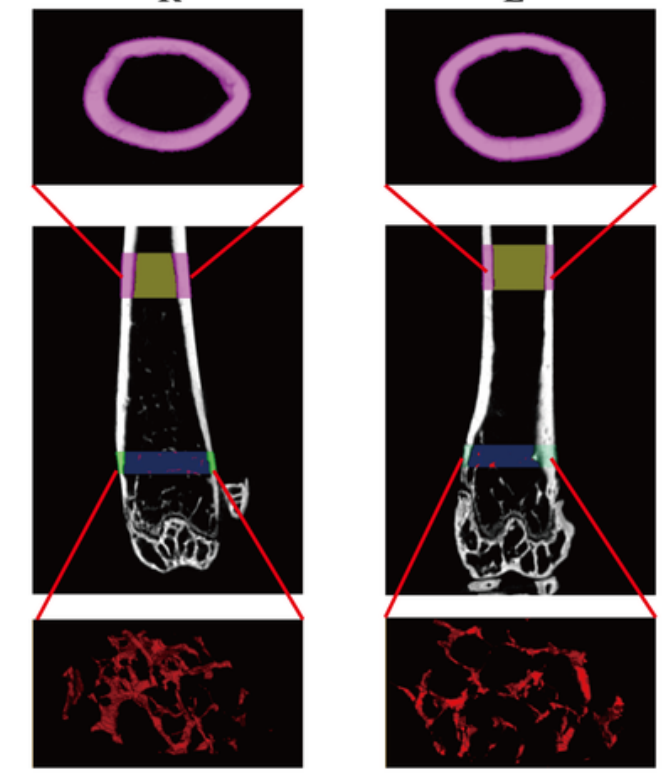

$* * * *$

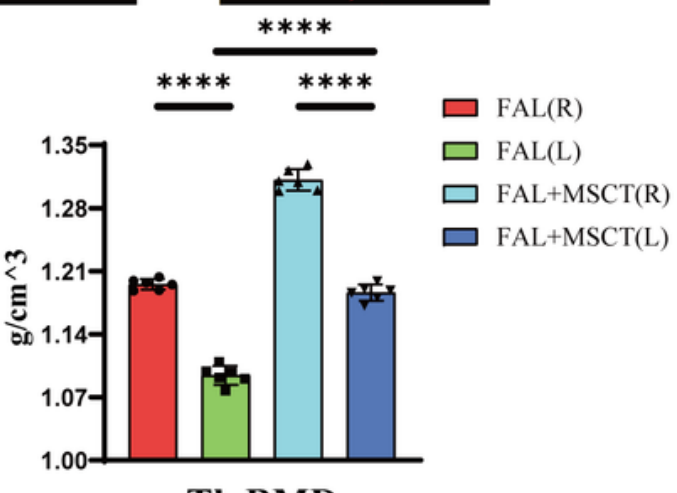

Tb.BMD

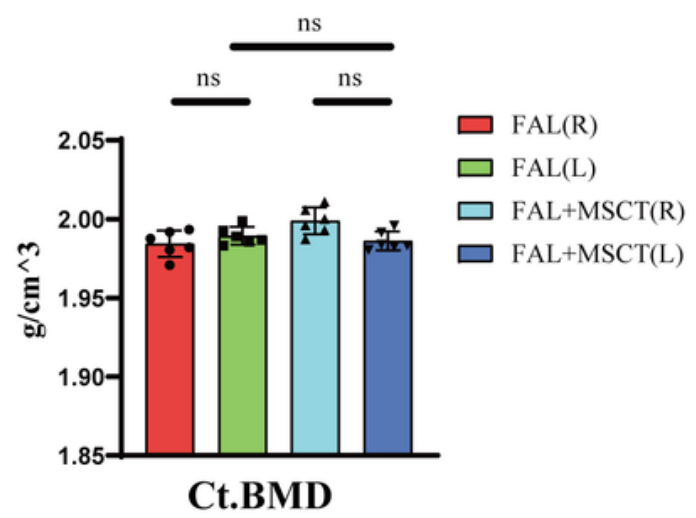

Figure 5

Prevention of femoral artery ligation (FAL)-induced osteoporosis in mice. (a) Micro-CT bone scan and CTbased three-dimensional microangiography of mouse femur on day 28 after FAL surgery (R: right side, L: 
left side). (b) Quantitative analysis of Tb .BV/TV, Tb.N, and Tb.BMD. (c) Quantitative analysis of Ct.BMD, Ct.Th, and Ct.Ar/Tt.Ar. $n=6$ per group. ${ }^{*} P<0.05,{ }^{*} P<0.01,{ }^{*} * * P<0.001,{ }^{*} * \star P<0.0001, n s \geq 0.05$.

A

Merge
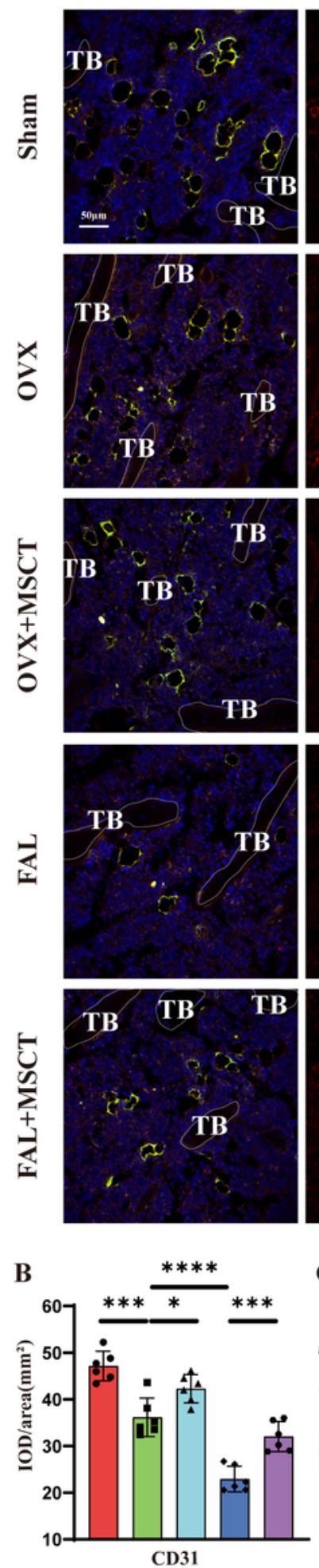

EMCN
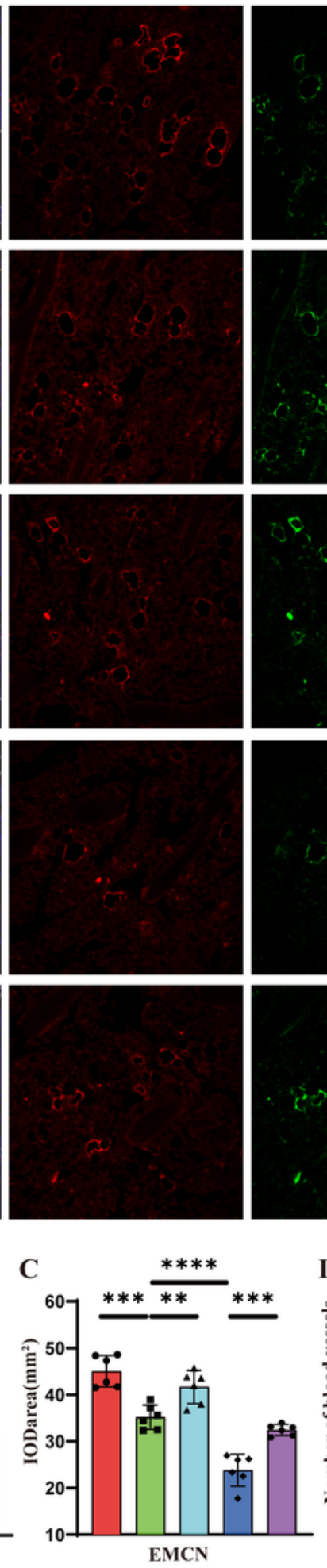

CD31
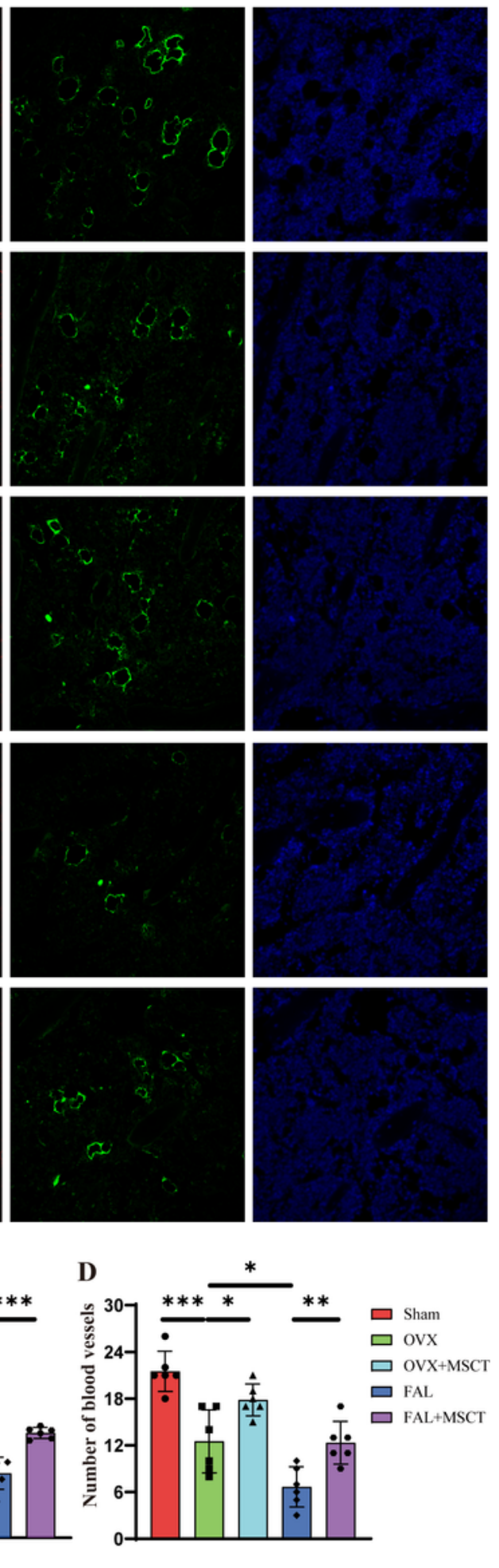

Figure 6

Cell transplantation promotes an increase in $\mathrm{H}$-type vessels in the femoral bone marrow cavity. (a) Immunofluorescence staining of paraffin sections of mouse femurs (CD31: green; EMCN: red; merge:

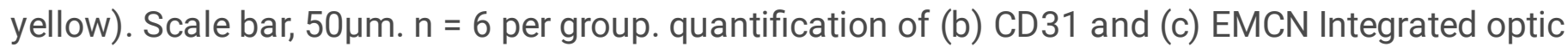


density. (d) Calculation of the number of blood vessels. $n=6$ per group. ${ }^{\star} P<0.05,{ }^{\star \star} P<0.01$, ${ }^{\star \star \star} P<$

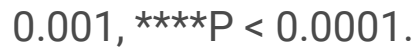

